\title{
The effect of luminance values of the edge between regions on figure-ground perception
}

\author{
Yukyu Araragi $^{1} \cdot$ Yu Sugimoto ${ }^{2}$
}

Published online: 26 February 2019

(C) The Psychonomic Society, Inc. 2019

\begin{abstract}
The present study examined whether luminance values of an edge between regions affect figure-ground perception. A dark-gray or light-gray region was presented on the left (or upper) or right (or lower) side of an edge. Observers reported which region was perceived as a figure. The results in the $0.2^{\circ}$ edge width condition showed that dark-gray regions with low luminance edges were perceived as a figure significantly more often than dark-gray regions with high luminance edges. The edge luminance effect occurred, partly occurred, and did not occur when the edge widths were $0.2,0.5$, and $0.8^{\circ}$ of visual angle, respectively. These results showed that luminance values of the edge between the regions affected figure-ground perception, and that regions with luminance values closer to that of the edge between the regions were perceived as a figure more often, but only when the edge width was narrow. The edge luminance effect occurred less often as the edge width was increased and would be attributed to similarity grouping between edge and region.
\end{abstract}

Keywords Figure-ground perception $\cdot$ Edge between regions $\cdot$ Luminance value $\cdot$ Width of edge $\cdot$ Edge assignment $\cdot$ Bipartite arrangement

\section{Introduction}

Figure-ground perception is a well-known phenomenon in perceptual psychology. Shaped entities are known as "figures," and the shapeless region that appears to continue behind the figures as background is known as "ground" (Peterson \& Salvagio, 2008). Generally, in a Rubin's face/ goblet pattern (Rubin, 1915/1958, see also Kawabe \& Miura, 2004; Takashima, Fujii, \& Shiina, 2012), which is an ambiguous figure pattern seen as either faces or goblet, if the faces are seen, the faces region is called figure and the goblet region is called ground. If the goblet is seen, the goblet region is called figure and the faces region is called ground.

Previous studies have reported internal surface features of figure regions for figure-ground perception (Ghose \& Palmer,

Yukyu Araragi

uqa@hmn.shimane-u.ac.jp

1 Institute of Human Sciences, Shimane University, 1060 Nishikawatsu-cho, Matsue-shi, Shimane 690-8504, Japan

2 Faculty of Law and Literature, Shimane University, Matsue-shi, Japan
2010, 2016; Hulleman \& Humphreys, 2004; Kanizsa \& Gerbino, 1976; Palmer \& Brooks, 2008; Seno, Ito, \& Sunaga, 2009; Vecera, Vogel, \& Woodman, 2002; Wake, Wake, \& Oyama, 2014; see also, Wagemans et al., 2012 for a review). For example, regions that are smaller rather than larger, convex rather than concave, positioned lower rather than upper in the stimulus, and having a wide base and narrow top rather than a narrow base and wide top, are perceived as a figure more often.

Edges between regions, which are an external feature for surfaces of figure regions, are important cues for figureground perception. Edges belong to the figure region (Peterson \& Enns, 2005; Peterson, Mojica, Salvagio, \& Kimchi, 2017; Seno et al., 2009). Moreover, Kawabe and Miura (2003) showed, using face/goblet patterns, that regions filled with top-lit disks were perceived as a figure less often when edges between the face and goblet regions were painted by black (i.e., when the edges were absent) than when the edges were present. The results indicated that the presence or absence of edges between the face and goblet regions affected figure-ground perception. Furthermore, it has been reported that similarity grouping between edge and region (Palmer \& Brooks, 2008) and border-ownership cells (Zhou, Freidman, \& von der Heydt, 2000) affect figure-ground perception. 
It has also been shown that differences in luminance between regions and background affect figure-ground perception. Oyama (1960) examined these differences using the stimuli that two regions consisted of six sectors with $60^{\circ}$ in a circle. The three sectors were white or black (i.e., fixed region). The backgrounds were black and white in the white and black fixed regions, respectively. The brightness of the other three sectors (i.e., variable region) was manipulated, and the edges between sectors were fixed black thin line segments. The results showed that the white region was perceived as a figure more often than the other region, when the other region (i.e., the variable region) had nearly the same brightness as the black background. The black region was perceived as a figure more often than the other region, when the other region had nearly the same brightness as the white background. The results indicated that the regions with luminance values further from that of the background were more often perceived as a figure (see also O'shea, Blackburn, \& Ono, 1994).

Luminance of the edge between regions could be an important cue for figure-ground perception. It is not fully clear that the edges between regions affect figure-ground perception, since there are few previous studies in which line segments of edges themselves were manipulated directly (Brooks \& Driver, 2010; Kawabe \& Miura, 2003; Palmer \& Brooks, 2008). Specifically, no previous study has systematically examined whether luminance values of the edge itself between regions affect figure-ground perception. ${ }^{1}$ If the luminance values of the edge between regions affect figure-ground perception (hereafter, edge luminance effect), then this effect has two possible tendencies: regions with luminance values closer to and further from that of the edge between regions were perceived as a figure more often. Similarity grouping between edge and region (Palmer \& Brooks, 2008) suggests that regions with luminance values closer to that of the edge between regions were perceived as a figure more often. On the other hand, the results in Oyama (1960) suggest that regions with luminance values further from that of the edge between regions might be perceived as a figure more often if the edge between regions is processed as a part of the background ( since the present study adopted a thick edge ${ }^{2}$ outside of a thin line, as shown in Fig. 1). In addition, the width of the edge might affect the (thick) edge processing that indicates whether the edge between regions is processed as just a part of the background or as the edge, an important cue for figure-

\footnotetext{
${ }^{1}$ Palmer and Brooks (2008) did not examine similarity grouping for luminance.

${ }^{2}$ Line edges with thickness might be less common in natural images than line edges of contrast boundaries without thickness. However, line edges with thickness are present in many human artifacts (Palmer \& Brooks, 2008) such as the rim of a mug, the rounded edges of safety posts, and white lines indicating traffic lanes on a road. Moreover, line edges with thickness are commonly found in 2-D drawings (Palmer \& Brooks, 2008). Depth perceptions for recent human artifacts (i.e., computer buttons on a computer display) reflect shading depth cues (Fujita, 2007). Line edges with thickness also would reflect universal human visual processing of edges.
}

ground perception. The edge luminance effect would suggest specified mechanistic relationships between edge assignment (i.e., border-ownership) and figure-ground perception.

The present study examined the effect of luminance values of the edge between regions on figure-ground perception. In the present study, to directly examine the effect of luminance values of the edge itself, we adopted thick edges with each of five luminance values, as shown in Fig. 1. To examine edge assignment directly, surface of region and edge were defined only by luminance values. Experiments 1 and 2 examined the effect of luminance values of the edge on figure-ground perception in the horizontal and vertical stimulus configurations, respectively. To explore what edge widths the visual processing in figure-ground perception regards as the edge of the figure, Experiment 3 examined the effect of the edge width ${ }^{2}$ $\left(0.2,0.5\right.$, or $0.8^{\circ}$ of visual angle $)$ on the edge luminance effect in the horizontal configuration. Experiment 4 examined the effect of the absence or presence of a $0.2^{\circ}$ edge on figureground perception.

\section{Experiment 1}

\section{Methods}

\section{Observers}

Twelve Japanese adults (19-32 years old: $M=20.9$ years old, $S D=3.6$; ten female, two male) participated as observers. They had normal or corrected-to-normal binocular visual acuity measured using a screenoscope (SS-3, Topcon). They were unaware of the purpose of the experiment. The experiment was performed with the written informed consent of each observer.

\section{Apparatus}

The stimuli were presented on a 22-in. CRT monitor (RDF223G, Mitsubishi). A computer (Vostro 220s, Dell) was used to control the presentation of the stimuli and to record responses that the observers made by pressing assigned keys. The observers' heads were held in position with a forehead and chin rest.

\section{Stimuli}

Figure 1 shows typical examples of stimuli used in Experiment 1. The stimuli were similar to those used in Ghose and Palmer (2010) with regard to shapes of regions, stimulus size, and the use of random-dot backgrounds. Regions that were assigned as figure or ground were defined by luminance as dark-gray or light-gray regions (hereafter, dark regions or light regions). Each region was presented on the left or right side of the edge. The luminances of the dark 
a
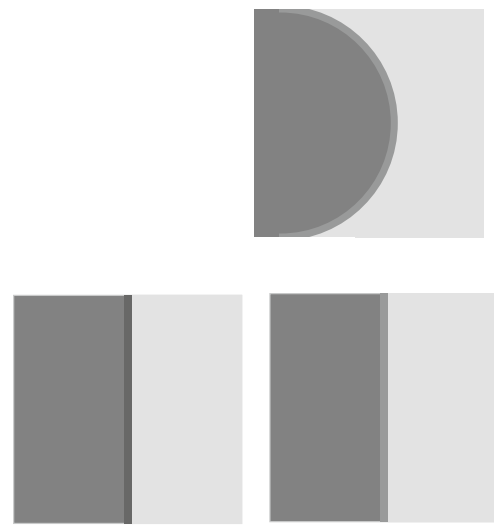

d

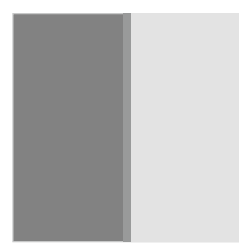

e b
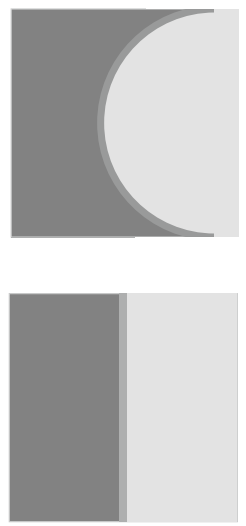

f

C

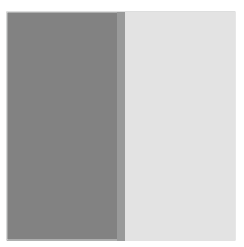

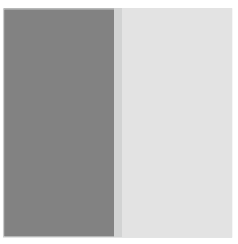

g

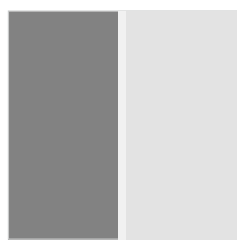

h
Fig. 1 Stimuli in Experiment 1. (a-c) There were three shapes of darkgray regions, convex, concave, and rectangular. (d-h) There were five luminance values $\left(5.9,20.5,33.3,42.9\right.$, or $\left.64.2 \mathrm{~cd} / \mathrm{m}^{2}\right)$ of the edge

and light regions were 12.1 and $52.1 \mathrm{~cd} / \mathrm{m}^{2}$, respectively. The shape of the dark or light region was convex, concave, or rectangular, as shown in Fig. 1. Dark and light regions had equal area. The width of the edge was $0.2^{\circ}$ of visual angle. The luminance value of the edge between the regions was 5.9, $20.5,33.3,42.9$, or $64.2 \mathrm{~cd} / \mathrm{m}^{2}$. A random-dot pattern was presented as a background in the whole display. The luminance of the random-dot background was $31.1 \mathrm{~cd} / \mathrm{m}^{2}$. The luminance of a square dot was 12.1 or $52.1 \mathrm{~cd} / \mathrm{m}^{2}$. Each dot subtended $0.05^{\circ}$ vertically $\times 0.05^{\circ}$ horizontally. Stimuli (dark and light regions and edge) subtended $6.5^{\circ}$ vertically $\times 6.5^{\circ}$ horizontally. The stimulus was presented until the observers responded by pressing assigned keys.

\section{Procedures}

The observers viewed the stimuli using binocular vision in a darkened room. The viewing distance was $114.6 \mathrm{~cm}$.

The observers were requested to fixate on the center of stimuli, to simultaneously view two (left and right) regions, and to report which one was seen (i.e., consciously perceived) as a figure. Left and right sides corresponded to the " 1 " and " 3 " keys on the numeric keypad, ${ }^{3}$ respectively, to avoid observers being confused by the discordance of the relative location between regions and keys. Before the experiment, figure and ground were explained to the observers using the example of a figure of a Rubin's face/goblet pattern. In addition, the observers were told that a figure region tended to be perceived to be closer than a ground region. The observers were requested to report based on their initial perception, even if the perceived figure region switched with further viewing. If both left and right regions were seen

\footnotetext{
3 There was no significance of anisotropy between left (or upper) and right (or lower) regions in Experiments 1 and 2 in the present study.
}

between the dark-gray $\left(12.1 \mathrm{~cd} / \mathrm{m}^{2}\right)$ and light-gray $\left(52.1 \mathrm{~cd} / \mathrm{m}^{2}\right)$ regions. The width of the edge was $0.2^{\circ}$ of visual angle. In the experiment, the stimuli were actually presented on a random-dot background

simultaneously (i.e., both regions were initially perceived as a figure), the observers were requested to report only the region that was first seen as the figure after further viewing. After further viewing, if both left and right regions were still seen simultaneously, the observers were requested to report which one was perceived as the closer region to the observer as the figure, that is, to use a depth cue similar to that in Ghose and Palmer (2010).

Each observer completed 90 experimental trials (5 luminance values of edge $\times 3$ shapes of dark region $\times$ 2 sides of dark region $\times 3$ repetitions). The observers were requested to take breaks after the 15 th, 30th, 45th, 60 th, and 75 th experimental trials by computerdisplayed instructions. Before the experimental trials, each observer performed 34 practice trials to become accustomed to the task and the darkened room. The order of the trials was randomized for each observer.

\section{Results}

Results of Experiment 1 are shown in Fig. 2. A three-way repeated measures ANOVA was performed on the percentage of trials in which the dark region was perceived as a figure with edge luminance (five values), dark region shape (three shapes), and dark region side (two sides) as main factors. The main effect of the edge luminance was significant $\left[F(4,44)=16.430, p<.001, \eta_{\mathrm{p}}{ }^{2}=.60\right]$. Multiple comparisons (Ryan's method) showed that dark regions with a darker edge of 5.9 or $20.5 \mathrm{~cd} / \mathrm{m}^{2}$ were perceived as a figure significantly more often than dark regions with a lighter edge of $33.3,42.9$, or $64.2 \mathrm{~cd} / \mathrm{m}^{2}$. The results showed the edge luminance effect in the present study. The main effect of the dark region shape was significant $\left[F(2,22)=7.750, p=.003, \eta_{\mathrm{p}}{ }^{2}=.41\right]$. 


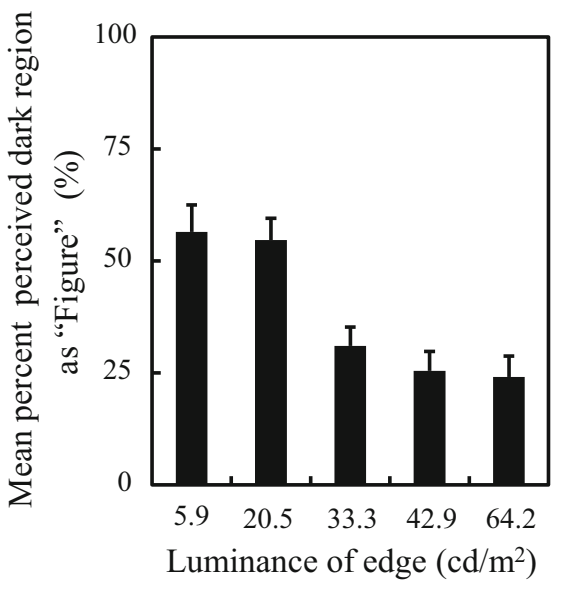

a

Fig. 2 Results of Experiment 1. Error bars represent standard errors. (a) Mean percentages of trials in which the dark region was perceived as a figure averaged over the 12 observers are plotted as a function of the

Convex regions were perceived as a figure significantly more often than concave or rectangular regions. ${ }^{4}$ The main effect of the dark region side was not significant $\left[F(1,11)=0.009, p=.93, \eta_{\mathrm{p}}^{2}=.001\right]$. The interaction between edge luminance and dark region shape, between edge luminance and dark region side, between dark region shape and dark region side, or between edge luminance, dark region shape, and dark region side was not significant $\left[F(8,88)=1.116, p=.36, \eta_{\mathrm{p}}^{2}=.09 ; F(4,44)=\right.$ $0.303, p=.87, \eta_{\mathrm{p}}{ }^{2}=.03 ; F(2,22)=0.953, p=.40, \eta_{\mathrm{p}}{ }^{2}$ $\left.=.08 ; F(8,88)=0.927, p=.50, \eta_{\mathrm{p}}^{2}=.08\right]$.

The results mainly showed the following two points. First, luminance values of the edge between the regions significantly affected figure-ground perception. Second, regions with luminance values closer to that of the edge were perceived as a figure more often. The edge luminance effect in the present study had a similar tendency to similarity grouping for some visual features ${ }^{1}$ between edge and region (Palmer \& Brooks, 2008).

\section{Experiment 2}

Experiment 2 examined the effect of luminance values of the edge between the regions on figure-ground perception in the vertical stimulus configuration.

\section{Methods}

The methods used in Experiment 2 were identical to those used in Experiment 1, except for the following points.

\footnotetext{
${ }^{4}$ The results in Experiments 1 and 2 supported the results for convex regions in Ghose and Palmer (2010) and in two region stimuli of Peterson and Salvagio (2008).
}

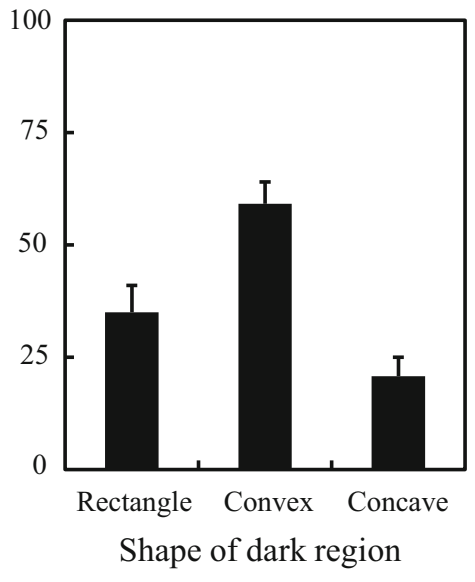

b

luminance of the edge. (b) Mean percentages of trials in which the dark region was perceived as a figure averaged over the 12 observers are plotted as a function of the shape of the dark region

Twelve Japanese adults (18-23 years old: $M=20.2$ years old, $S D=1.6$; four female, eight male) participated as observers. The dark or light region was presented on the upper or lower side of the edge, as shown in Fig. 3. The observers were requested to simultaneously view two (upper and lower) regions, and to report which one was seen (i.e., consciously perceived) as a figure. Upper and lower sides corresponded to " 1 " and " 3 " keys on the numeric keypad, respectively. ${ }^{3}$

\section{Results}

Results of Experiment 2 are shown in Fig. 4. A three-way repeated measures ANOVA was performed on the percentage of trials in which the dark region was perceived as a figure with edge luminance (five values), dark region shape (three shapes), and dark region side (two sides) as main factors. The main effect of the edge luminance was significant $[F(4,44)=$ 12.452, $\left.p<.001, \eta_{\mathrm{p}}^{2}=.53\right]$. Multiple comparisons showed that dark regions with a darker edge of 5.9 or $20.5 \mathrm{~cd} / \mathrm{m}^{2}$ were perceived as a figure significantly more often than dark regions with a lighter edge of $33.3,42.9$, or $64.2 \mathrm{~cd} / \mathrm{m}^{2}$. The results supported the edge luminance effect in Experiment 1. The main effect of the dark region shape was significant $[F(2$, 22) $\left.=16.414, p<.001, \eta_{\mathrm{p}}{ }^{2}=.60\right]$. Convex regions were perceived as a figure significantly more often than concave or rectangular regions. ${ }^{3}$ Rectangular regions were perceived as a figure significantly more often than concave regions. The main effect of the dark region side was not significant $[F(1$, 11) $\left.=3.988, p=.07, \eta_{\mathrm{p}}{ }^{2}=.27\right]$. The interaction between edge luminance and dark region shape, between edge luminance and dark region side, between dark region shape and dark region side, or between edge luminance, dark region shape, and dark region side was not significant $[F(8,88)=0.658, p=$ 

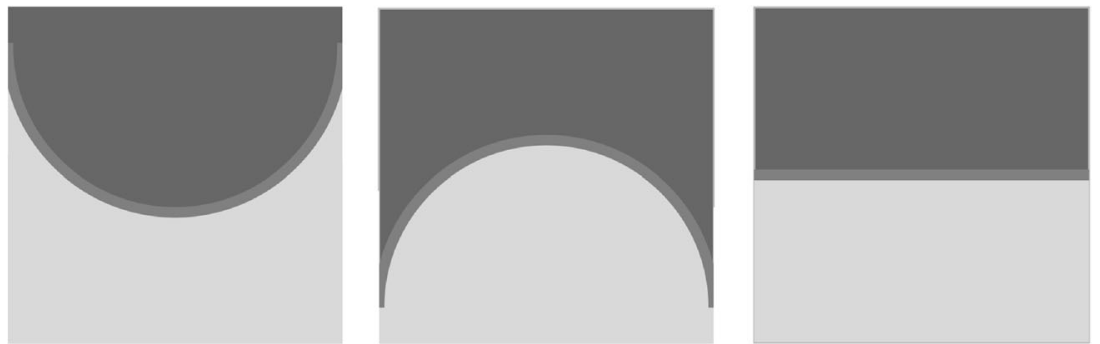

Fig. 3 Stimuli in Experiment 2. There were three shapes of dark regions - convex, concave, and rectangular. In the experiment, the stimuli were actually presented on a random-dot background

$.73, \eta_{\mathrm{p}}^{2}=.06 ; F(4,44)=0.679, p=.61, \eta_{\mathrm{p}}^{2}=.06 ; F(2,22)=$ $\left.0.580, p=.57, \eta_{\mathrm{p}}{ }^{2}=.05 ; F(8,88)=0.767, p=.63, \eta_{\mathrm{p}}{ }^{2}=.07\right]$.

The results mainly showed the following two points. First, in the vertical configuration, luminance values of the edge between regions also affected figure-ground perception: regions with luminance values closer to that of the edge were perceived as a figure more often. Second, there was no anisotropy between upper and lower regions that were perceived as a figure. The results suggest that the edge luminance effect of figure-ground perception was larger than the effect of the lower field cue in the present experimental condition.

\section{Experiment 3}

Experiments 1 and 2 showed the edge luminance effect of figure-ground perception that regions with luminance values closer to that of the edge were perceived as a figure more often. To explore what edge widths the visual processing in figure-ground perception regard as the edge of the figure, we examined the effect of the edge width on the edge luminance effect of figure-ground perception. To have equal familiarity with convex and concave shapes and to control edge width easily, the Japanese kanji characters for convex “凸” and concave “凹" 5 " were used as the convex and concave stimuli, respectively. Horizontal configuration was adopted to avoid the effects of the top-bottom polarity cue (Hulleman \& Humphreys, 2004) and the lower field cue (Vecera et al., 2002) on figure-ground perception.

\section{Methods}

The methods used in Experiment 3 were identical to those used in Experiment 1, except for the following points. Thirty-six Japanese adults (18-25 years old: $M=19.6$ years old, $S D=1.6 ; 20$ female, 12 male) participated as observers. The observers were divided into three groups according to the width of the edge between the regions. The stimuli were presented on a 21-in. CRT monitor (FlexScan T966, Eizo). The

\footnotetext{
${ }^{5}$ The “凹” shape was similar to the C-shaped figures used in Zhou et al. (2000).
}

“凸 and “" $^{5}$ were presented as the convex and concave shapes of dark or light regions, respectively, as shown in Fig. 5. The width of the edge between regions was $0.2,0.5$, or $0.8^{\circ}$ of visual angle. The luminances of the dark and light regions were 12.0 and $51.6 \mathrm{~cd} / \mathrm{m}^{2}$, respectively. The luminance value of the edge between regions was 5.8, 20.4, 33.2, 42.8 , or $64.3 \mathrm{~cd} / \mathrm{m}^{2}$. The luminance of the random-dot background was $31.9 \mathrm{~cd} / \mathrm{m}^{2}$. The luminance of a dot was 12.0 or $51.6 \mathrm{~cd} / \mathrm{m}^{2}$. Stimuli subtended $6.6^{\circ}$ vertically $\times 6.6^{\circ}$ horizontally. Each observer completed 60 experimental trials ( 5 luminance values of edge $\times 3$ shapes of dark region $\times 2$ sides of dark region $\times 2$ repetitions). Before the experimental trials, each observer performed 33 practice trials to become accustomed to the task and the darkened room. The observers were requested to simultaneously view two (left and right) regions, and to report which one was perceived as shaped or was seen (i.e., consciously perceived) as a figure. In addition, the observers were told that figure regions have shape, and ground regions are perceived as background. The depth cue instruction used in Experiments 1 and 2 was not adopted in Experiment 3. The observers were requested to take breaks after the 15 th, 30 th, and 45 th experimental trials by computerdisplayed instructions.

\section{Results and discussion}

Results of Experiment 3 are shown in Fig. 6. A three-way mixed ANOVA was performed on the percentage of trials in which the dark region was perceived as a figure with edge width (three widths), edge luminance (five values), and dark region shape (three shapes) as main factors. The main effect of the edge width was not significant $[F(2,33)=2.575, p=.09$, $\left.\eta_{\mathrm{p}}{ }^{2}=.14\right]$. The main effect of the edge luminance was significant $\left[F(4,132)=14.128, p<.001, \eta_{\mathrm{p}}{ }^{2}=.30\right]$. Multiple comparisons showed that dark regions with a darker edge of 5.8 or $20.4 \mathrm{~cd} / \mathrm{m}^{2}$ were perceived as a figure significantly more often than dark regions with a lighter edge of $33.2,42.8$, or $64.3 \mathrm{~cd} /$ $\mathrm{m}^{2}$. The main effect of the dark region shape was not significant $\left[F(2,66)=0.097, p=.91, \eta_{\mathrm{p}}{ }^{2}=.00\right]$. The interaction between edge width and edge luminance was significant $\left[F(8,132)=2.469, p=.016, \eta_{\mathrm{p}}{ }^{2}=.13\right]$. In the 0.2 and $0.5^{\circ}$ edge conditions, the simple main effect of the edge luminance 


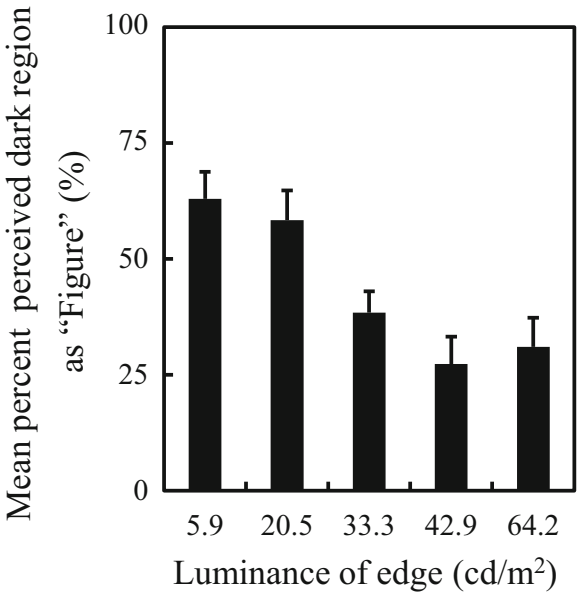

a

Fig. 4 Results of Experiment 2. Error bars represent standard errors. (a) Mean percentages of trials in which the dark region was perceived as a figure averaged over the 12 observers are plotted as a function of the

was significant, whereas in the $0.8^{\circ}$ edge condition, the simple main effect of the edge luminance was not significant $\left[0.2^{\circ}: F(4,132)=8.895, p<.001 ; 0.5^{\circ}: F(4,132)\right.$ $\left.=9.180, p<.001 ; 0.8^{\circ}: F(4,132)=0.992, p=.41\right]$. In the $0.2^{\circ}$ edge condition, multiple comparisons showed that dark regions with a darker edge of 5.8 or $20.4 \mathrm{~cd} / \mathrm{m}^{2}$ were perceived as a figure significantly more often than dark regions with a lighter edge of $33.2,42.8$, or $64.3 \mathrm{~cd} / \mathrm{m}^{2}$. The results supported the edge luminance effect in Experiments 1 and 2 . In the $0.5^{\circ}$ edge condition, multiple

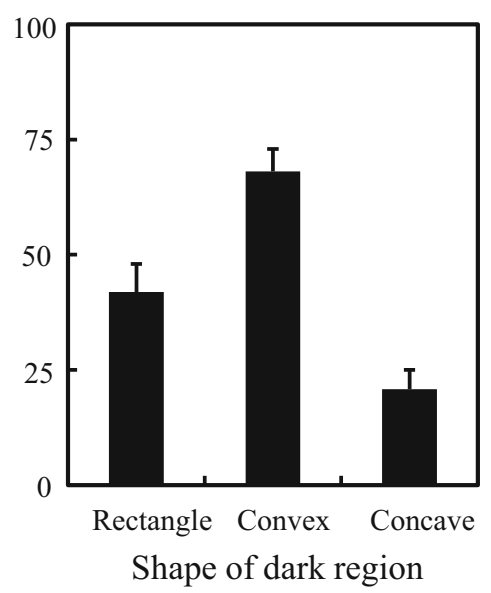

b

luminance of the edge. (b) Mean percentages of trials in which the dark region was perceived as a figure averaged over the 12 observers are plotted as a function of the shape of the dark region

comparisons showed that dark regions with a darker edge of $5.8 \mathrm{~cd} / \mathrm{m}^{2}$ were perceived as a figure significantly more often than dark regions with a lighter edge of 33.2, 42.8, or $64.3 \mathrm{~cd} / \mathrm{m}^{2}$. The results partly supported the edge luminance effect in the present study. The results in the $0.8^{\circ}$ edge condition did not support the edge luminance effect in the present study. In the 5.8, 20.4 , or $33.2 \mathrm{~cd} / \mathrm{m}^{2}$ edge condition, the simple main effect of the edge width was not significant, whereas in the 42.8 and $64.3 \mathrm{~cd} / \mathrm{m}^{2}$ edge conditions, the simple main effect of

a
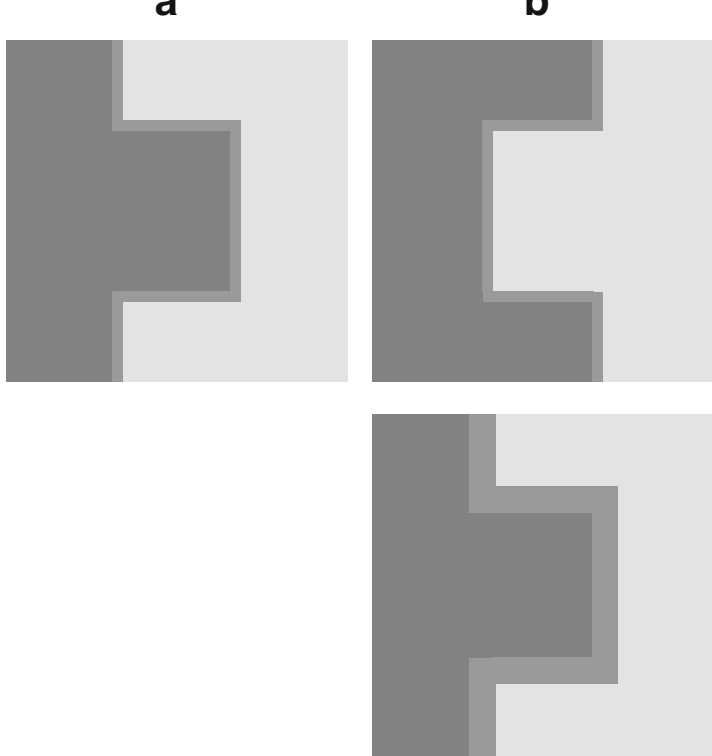

d
C
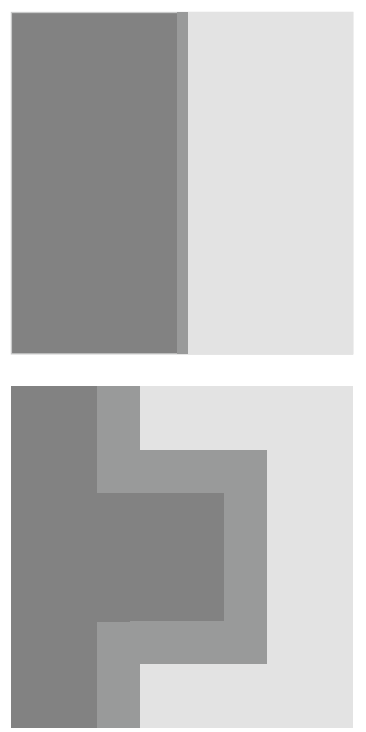

e
Fig. 5 Stimuli in Experiment 3. (a-c) Examples of stimuli in the $0.2^{\circ}$ edge width condition. The three shapes of the dark region were convex, concave, and rectangular. (a, d, e) Examples of stimuli in which dark regions were convex. There were three widths of the edge between the regions, $0.2,0.5$, and $0.8^{\circ}$ of visual angle. In the experiment, the stimuli were actually presented on a random-dot background 


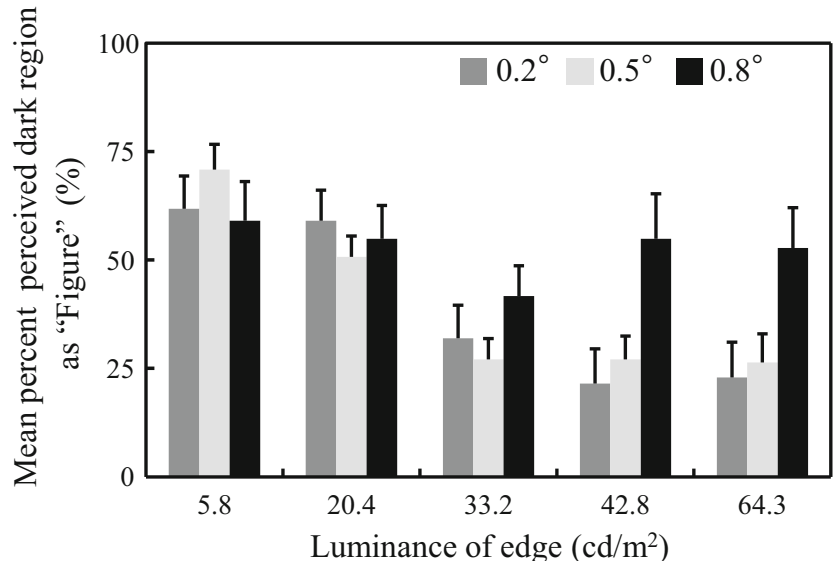

Fig. 6 Results of Experiment 3. Error bars represent standard errors. Mean percentages of trials that the dark region was perceived as a figure averaged over the 12 observers are plotted as a function of the luminance of the edge, separately for each width of the edge

the edge width was significant $\left[5.8 \mathrm{~cd} / \mathrm{m}^{2}: F(2,165)=\right.$ $0.687, p=.50 ; 20.4 \mathrm{~cd} / \mathrm{m}^{2}: F(2,165)=0.313, p=.73$; $33.2 \mathrm{~cd} / \mathrm{m}^{2}: F(2,165)=0.994, p=.37 ; 42.8 \mathrm{~cd} / \mathrm{m}^{2}: F(2$, $165)=5.747, p=.004 ; 64.3 \mathrm{~cd} / \mathrm{m}^{2}: F(2,165)=4.806, p=$ $.009]$. In the 42.8 and $64.3 \mathrm{~cd} / \mathrm{m}^{2}$ edge luminance conditions, multiple comparisons showed that dark regions with $0.8^{\circ}$ edge width were perceived as a figure significantly more often than dark regions with 0.2 or $0.5^{\circ}$ edge width. The interaction between edge width and dark region shape or between edge width, edge luminance, and dark region shape was not significant $\left[F(4,66)=0.635, p=.64, \eta_{\mathrm{p}}{ }^{2}=\right.$ $\left..04 ; F(16,264)=0.557, p=.91, \eta_{\mathrm{p}}{ }^{2}=.03\right]$. The interaction between edge luminance and dark region shape was significant $\left[F(8,264)=2.285, p=.022, \eta_{\mathrm{p}}{ }^{2}=.07\right]$. In all dark region shape conditions, the simple main effect of the edge luminance was significant. [rectangular: $F(4$, $396)=15.173, p<.001$; convex: $F(4,396)=6.692, p<$ .001 ; concave: $F(4,396)=7.109, p<.001]$. In the 5.8 , $33.2,42.8$, or $64.3 \mathrm{~cd} / \mathrm{m}^{2}$ edge condition, the simple main effect of the edge luminance was not significant, whereas in the $20.4 \mathrm{~cd} / \mathrm{m}^{2}$ edge condition, the simple main effect of the dark region shape was significant $\left[5.8 \mathrm{~cd} / \mathrm{m}^{2}: F(2\right.$, $330)=0.057, p=.94 ; 20.4 \mathrm{~cd} / \mathrm{m}^{2}: F(2,330)=3.781, p=$ $.024 ; 33.2 \mathrm{~cd} / \mathrm{m}^{2}: F(2,330)=0.424, p=.65 ; 42.8 \mathrm{~cd} / \mathrm{m}^{2}$ : $F(2,330)=0.652, p=.52 ; 64.3 \mathrm{~cd} / \mathrm{m}^{2}: F(2,330)=0.076$, $p=.93]$. In the rectangular and concave conditions, the multiple comparisons showed that dark regions with a darker edge of 5.8 or $20.4 \mathrm{~cd} / \mathrm{m}^{2}$ were perceived as a figure significantly more often than dark regions with a lighter edge of $33.2,42.8$, or $64.3 \mathrm{~cd} / \mathrm{m}^{2}$. In the convex condition, the multiple comparisons showed that dark regions with a darker edge of $5.8 \mathrm{~cd} / \mathrm{m}^{2}$ were perceived as a figure significantly more often than dark regions with a lighter edge of 20.4, $33.2,42.8$, or $64.3 \mathrm{~cd} / \mathrm{m}^{2}$. In the $20.4 \mathrm{~cd} / \mathrm{m}^{2}$ edge condition, the multiple comparisons showed that dark regions with a rectangular shape were perceived as a figure significantly more often than dark regions with a convex shape.

The results mainly showed that the edge luminance effect occurred less often as the edge width was increased. The results showed that the width of the edge between regions affected the edge luminance effect of figure-ground perception. Visual processing in figure-ground perception would regard an edge of less than $0.5^{\circ}$ as the edge of a figure and would show a limit of the similarity grouping between edge and region. The edge width coincided with that of $0.15^{\circ}$ in Palmer and Brooks (2008).

On the other hand, the results of Experiments 1, 2, and 3 suggest that light regions might be perceived as a figure more often than dark regions (hereafter, the light region bias). Light regions with the middle luminance edge (i.e., 33.3 or $33.2 \mathrm{~cd} /$ $\mathrm{m}^{2}$ ) were perceived as a figure significantly more often than dark regions with the middle luminance edge when the edge width was $0.2^{\circ}$. Therefore, we conducted Experiment 4 to explore the effect of light region bias on the edge luminance effect found in the present study.

\section{Experiment 4}

Experiments 1,2, and 3 showed the edge luminance effect of figure-ground perception that regions with luminance values closer to that of the edge were perceived as a figure more often when the edge width was narrow. On the other hand, the results suggested a light region bias that the light region might be perceived as a figure more often than dark region. To explore the effect of light region bias on the edge luminance effect, we examined the effect of the absence or presence of a $0.2^{\circ}$ edge on figure-ground perception. Stimuli included $0.2^{\circ}$ edges with four luminances or did not include the $0.2^{\circ}$ edge.

\section{Methods}

The methods used in Experiment 4 were identical to those used in Experiment 3, except for the following points. Eighteen Japanese adults (18-23 years old: $M=19.2$ years old, $S D=1.6 ; 7$ female, 11 male) participated as observers and performed all conditions. New shape stimuli were presented as dark or light regions as shown in Fig. 7 in order that the fixation point (i.e., the center of stimuli) was not located on a region and to decrease the effect of attention on figure-ground perception. The width of the edge between regions was either $0.2^{\circ}$ of visual angle or there was no $0.2^{\circ}$ edge. The luminances of the dark and light regions were 12.1 and $52.0 \mathrm{~cd} / \mathrm{m}^{2}$, respectively. The luminance value of the edge between regions was $5.8,20.6,43.0$, or $64.6 \mathrm{~cd} / \mathrm{m}^{2}$. The luminance of the random-dot background was $31.9 \mathrm{~cd} / \mathrm{m}^{2}$. The luminance of a dot was 12.1 or $52.0 \mathrm{~cd} / \mathrm{m}^{2}$. In the experiment, a 
a

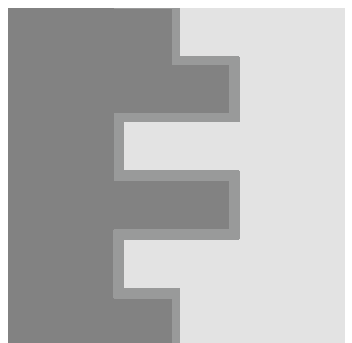

b

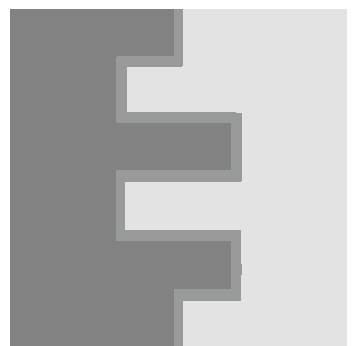

C

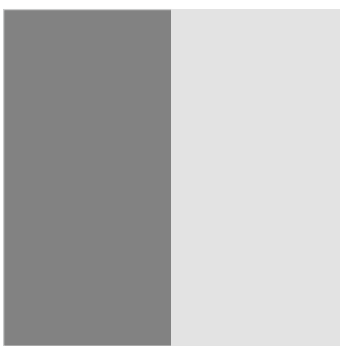

Fig. 7 Stimuli in Experiment 4. (a, b) Examples of new shapes of a region. (c) Examples of stimuli with no $0.2^{\circ}$ edge. In the experiment, the stimuli were actually presented on a random-dot background

display of random-dot patterns with gray fixation cross was presented before the new shape stimuli display in order to decrease the effect of attention on figure-ground perception. After a response key was pressed, random-dot patterns $(3,000 \mathrm{~ms})$, gray background $(2,000 \mathrm{~ms})$, and random-dot patterns $(2,000 \mathrm{~ms})$ were presented to avoid the effect of afterimages on figure-ground perception. The luminance of the gray fixation cross or gray background was $33.4 \mathrm{~cd} / \mathrm{m}^{2}$. Before the experimental trials, each observer performed 24 practice trials to become accustomed to the task and the darkened room. After the further viewing, if both left and right regions were simultaneously perceived as a figure or if neither left nor right region was perceived as a figure, the observers were requested to press the "9" key on the numeric keypad. The observers were requested to take breaks after the 12th, 24th, 36th, and 48th experimental trials by computerdisplayed instructions.

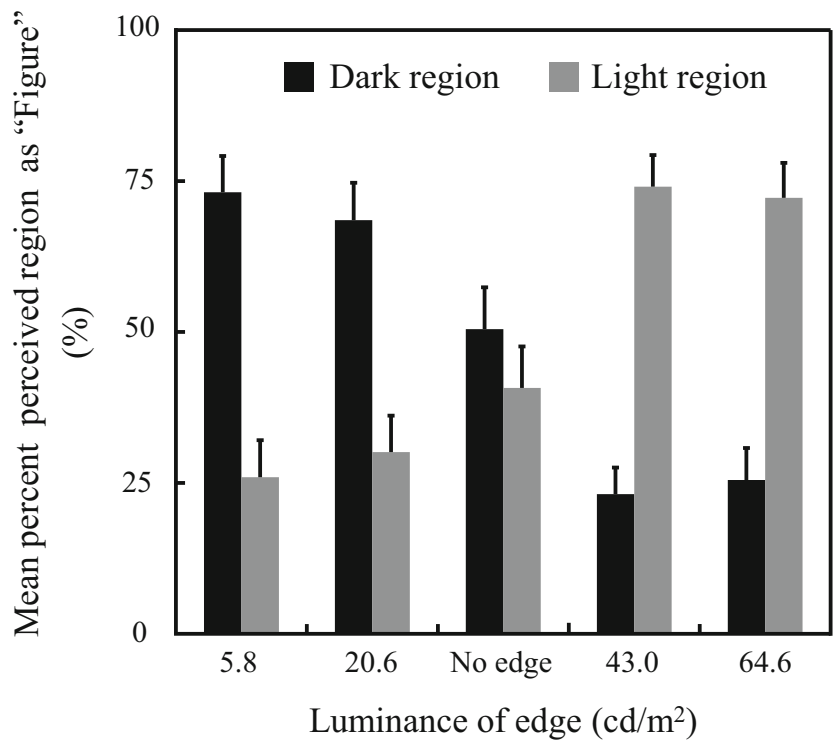

Fig. 8 Results of Experiment 4. Error bars represent standard errors. Mean percentages of trials in which the dark or light region was perceived as a figure averaged over the 18 observers are plotted as a function of the luminance of the edge, separately for the figural region

\section{Results and discussion}

Results of Experiment 4 are shown in Fig. $8 .^{6}$ A two-way repeated ANOVA was performed on the percentage of trials in which the dark or light region was perceived as a figure with edge luminance (four values + no edge) and figural region (two regions) as main factors. The main effect of the edge luminance was significant $\left[F(4,68)=2.902, p=.03, \eta_{\mathrm{p}}{ }^{2}=\right.$ .15]. The multiple comparisons showed that regions with a darker edge of $5.8 \mathrm{~cd} / \mathrm{m}^{2}$ were perceived as a figure significantly more often than regions with no edge. The main effect of the figural region was not significant $[F(1,17)=0.003, p=$ $\left..96, \eta_{\mathrm{p}}{ }^{2}=.00\right]$. The interaction between edge luminance and figural region was significant $\left[F(4,68)=27.936, p<.001, \eta_{\mathrm{p}}{ }^{2}\right.$ $=.62]$. In both figural regions, the simple main effect of the edge luminance was significant [dark region: $F(4,136)=$ 27.213, $p<.001$; light region: $F(4,136)=26.471, p<.001]$. In the dark region, multiple comparisons showed that dark regions with a darker edge of 5.8 or $20.6 \mathrm{~cd} / \mathrm{m}^{2}$ were perceived as a figure significantly more often than dark regions with no edge or a lighter edge of 43.0 or $64.6 \mathrm{~cd} / \mathrm{m}^{2}$. Dark regions with no edge were perceived as a figure significantly more often than dark regions with a lighter edge of 43.0 or $64.6 \mathrm{~cd} / \mathrm{m}^{2}$. In the light region, multiple comparisons showed that light regions with a lighter edge of 43.0 or $64.6 \mathrm{~cd} / \mathrm{m}^{2}$ were perceived as a figure significantly more often than light regions with no edge or a darker edge of 5.8 or $20.6 \mathrm{~cd} / \mathrm{m}^{2}$. In the $5.8,20.6$, 43.0 and $64.6 \mathrm{~cd} / \mathrm{m}^{2}$ edge conditions, the simple main effect of the figural region was significant, whereas in the no edge condition, the simple main effect of the figural region was not significant $\left[5.8 \mathrm{~cd} / \mathrm{m}^{2}: F(1,85)=16.350, p<.001 ; 20.6 \mathrm{~cd} /\right.$ $\mathrm{m}^{2}: F(1,85)=10.826, p=.002$; no edge: $F(1,85)=0.693, p=$ $.41 ; 43.0 \mathrm{~cd} / \mathrm{m}^{2}: F(1,85)=19.016, p<.001 ; 64.6 \mathrm{~cd} / \mathrm{m}^{2}: F(1$, $85)=16.031, p<.001]$. That is, the dark regions with a darker edge of 5.8 and $20.6 \mathrm{~cd} / \mathrm{m}^{2}$ were perceived as a figure significantly more often than light regions with a darker edge of 5.8

\footnotetext{
${ }^{6}$ The mean percentage of trials in which both dark and light regions were simultaneously perceived as a figure and neither dark nor light region was perceived as a figure (i.e., pressed the "9" key) averaged over five edge luminance conditions was $3.2 \%$. The mean percentage in no edge condition was $8.8 \%$.
} 
and $20.6 \mathrm{~cd} / \mathrm{m}^{2}$, respectively. The light regions with a lighter edge of 43.0 and $64.6 \mathrm{~cd} / \mathrm{m}^{2}$ were perceived as a figure significantly more often than dark regions with a lighter edge of 43.0 and $64.6 \mathrm{~cd} / \mathrm{m}^{2}$, respectively. These results support the edge luminance effect in the present study. There was no significant difference between dark and light regions with no $0.2^{\circ}$ edge. The results did not support the light region bias.

The results mainly showed the following two points. First, regions with luminance values closer to that of the edge were perceived as a figure more often than regions with no $0.2^{\circ}$ edge. Second, there was no bias between dark and light regions that were perceived as a figure. The results showed that the edge luminance effect itself was not attributed to the light region bias and the light region bias was not robust.

\section{General discussion}

The present study showed the edge luminance effect in figureground perception that regions with luminance values closer to that of the edge between the regions were perceived as a figure more often, but only when the edge width was narrow. Experiments 1 and 2 showed that dark-gray regions with low luminance edges were perceived as a figure significantly more often than dark-gray regions with high luminance edges, both in the horizontal and in the vertical stimulus configurations. These results also showed that light-gray regions with high luminance edges were perceived as a figure significantly more often than light-gray regions with low luminance edges. Experiment 3 showed that the edge luminance effect occurred, partly occurred, and did not occur when the edge width was $0.2,0.5$, and $0.8^{\circ}$ of visual angle, respectively. The results showed that the edge luminance effect occurred less often as the edge width was increased. Experiment 4 showed that darkgray regions with low luminance edges were perceived as a figure significantly more often than dark-gray regions with no $0.2^{\circ}$ edges and light-gray regions with high luminance edges were perceived as a figure significantly more often than lightgray regions with no $0.2^{\circ}$ edges.

The edge luminance effect would be attributed to similarity grouping between edge and region. The results in the $0.2^{\circ}$ edge width condition showed that regions with luminance values closer to that of the edge between the regions were perceived as a figure more often. The edge luminance effect in the present study had similar tendency to similarity grouping of some visual features ${ }^{1}$ between edge and region (Palmer $\&$ Brooks, 2008). The present study directly showed grouping between edge and surface of region, since edge and surface of region were defined by only luminance values. The results in the $0.8^{\circ}$ edge width condition would show a limit of grouping between edge and region. The results in the present study added the grouping of luminance information to similarity grouping between edge and region in Palmer and Brooks (2008).

The edge luminance effect was unlikely to be attributed to shading cues. For example, when an object (such as a cuboid) is lit from above, the luminance of the top of the object is higher than that of the sides. When the region and edge in the present experiments are thought to correspond to the top and side of an object, respectively, as similar to extremal edges (Ghose \& Palmer, 2010), the top or light region is physically closer than the side, dark region, or darker edge than a figural region. The shading depth cue could account for the results for the darker edges than a figural region (e.g. 5.9 or $42.9 \mathrm{~cd} / \mathrm{m}^{2}$ edge in Experiment 1), whereas it could not account for the results for the lighter edges than a figural region (e.g. 20.5 or $64.2 \mathrm{~cd} / \mathrm{m}^{2}$ edge in Experiment 1). In addition, shading cues might partly affect the edge luminance effect in some conditions for the $0.5^{\circ}$ edge of Experiment 3 . That is, the edge luminance effect occurred with the edge of $5.8 \mathrm{~cd} / \mathrm{m}^{2}$, but not with $20.4 \mathrm{~cd} / \mathrm{m}^{2}$. On the other hand, the shading cues cannot account for the results that dark-gray regions with the 5.8 and $42.8 \mathrm{~cd} / \mathrm{m}^{2}$ edges were not perceived as a figure significantly more often than dark-gray regions with the 20.4 and $64.3 \mathrm{~cd} / \mathrm{m}^{2}$ edges, respectively. Moreover, in Experiment 2, there was no significant difference between upper and lower sides of dark or light region. Since the 2-D stimuli in the present study would be weak or no shading depth cues, they were unlikely to affect the edge luminance effect. On the other hand, strong shading depth cues in the 3-D rendering stimuli in Ghose and Palmer (2010) would affect figure-ground perception.

The results in the present study did not show the phenomenon that regions with luminance values further from that of the edge between regions were perceived as a figure more often. Even in the $0.8^{\circ}$ edge width condition of Experiment 3 , the phenomenon did not occur, although the edge between regions would be regarded as background. The luminance of the $0.8^{\circ}$ edge would have little or no effect as background, since the random-dot backgrounds were presented and the edge between regions was only one part of edges of a region, in the present study.

The absence of the edge luminance effect with the $0.8^{\circ}$ edge in Experiment 3 might be attributed to the parallelism cue (parallel edges). If only edge was perceived as a figure and edge was perceived as an independent region by the parallelism cue (parallel edges), then the edge luminance effect might not occur. Ren, Fowlkes, and Malik (2006) reported that the parallelism cue was useful for figure-ground perception. We conducted Informal Observations with four observers. The methods were similar to those with $0.8^{\circ}$ edges used in Experiment 3, except that the observers' task was to report only one region of left, central, or right regions that was perceived as a figure. The task was a three-alternative forced choice task. The results showed that the mean percentages, 
averaged over four edge luminance conditions, that the central region (i.e., the $0.8^{\circ}$ edges) and the outer two regions were perceived as a figure were $24 \%$ and $76 \%$, respectively. The percentage $(76 \%)$ of the outer two regions was more than the percentage (24\%) of the central region (i.e., the $0.8^{\circ}$ edge). On the other hand, $24 \%$ of trials might affect the absence of the edge luminance effect with the $0.8^{\circ}$ edge. On the other hand, the edge luminance effect with $0.2^{\circ}$ edges was not attributed to the parallelism cue. If only edge was perceived as a figure, then there was no significant difference between two regions (i.e., chance level). However, the results with $0.2^{\circ}$ edges in Experiments 1-4 showed the significant differences between two regions, based on the edge luminance effect. Moreover, the results in Experiment 4 showed that the mean percentage of trials that both left and right regions were simultaneously perceived as figures and neither left nor right region was perceived as a figure, averaged over the four edge luminance conditions, was $1.9 \%$. The results indicated that only $0.2^{\circ}$ edges were perceived as a figure in less than $1.9 \%$ of trials.

In summary, the present study showed the edge luminance effect of the luminance values that the edge between the regions affected figure-ground perception and that regions with the luminance values closer to that of the edge were perceived as a figure more often when the edge width was $0.2^{\circ}$. The edge luminance effect partly occurred when it was $0.5^{\circ}$, but not when it was $0.8^{\circ}$. That is, the edge luminance effect occurred less often as the edge width was increased. The results suggest that visual processing in figure-ground perception would regard an edge of less than $0.5^{\circ}$ as the edge of a figure and would show a limit of the similarity grouping between edge and region. The edge luminance effect directly showed the edge assignment and would be attributed to similarity grouping between edge and region.

Acknowledgements We wish to thank Miyuki Toga, Ai Nakashima, Yumi Oda, Masami Shimizu, and Haruka Sasaki for their insightful comments on the present study. We wish to thank reviewers for their insightful comments on an earlier manuscript of the present study.

Publisher's note Springer Nature remains neutral with regard to jurisdictional claims in published maps and institutional affiliations.

\section{References}

Brooks, J. L, \& Driver, J. (2010). Grouping puts figure-ground assignment in context by constraining propagation of edge assignment. Attention, Perception, \& Psychophysics, 72, 1053-1069.

Fujita, I. (2007). What it means to see ? Dojin Sensho. (In Japanese).
Ghose, T., \& Palmer, S. E. (2010). Extremal edges versus other principles of figure-ground organization. Journal of Vision, 10, 1-17.

Ghose, T., \& Palmer, S. E. (2016). Gradient cut and extremal edges in relative depth and figure-ground perception. Attention, Perception, \& Psychophysics, 78, 636-646.

Hulleman, J., \& Humphreys, G. W. (2004). A new cue to figure-ground coding: Top-bottom polarity. Vision Research, 44, 2779-2791.

Kanizsa, G., \& Gerbino, W. (1976). Convexity and symmetry in figureground organization. In M. Henle (Ed.), Vision and Artifact. (pp. 2532.) New York: Springer.

Kawabe, T., \& Miura, K. (2003). Figure-ground assignment based on shading. Japanese Journal of Psychonomic Science, 22, 10-17. (In Japanese with English abstract)

Kawabe, T., \& Miura, K. (2004). Perceptual grouping in shape from shading. Perception, 33, 601-614.

O'shea, R. P., Blackburn, S.G., \& Ono, H. (1994). Contrast as a depth cue. Vision Research, 34, 1595-1604.

Oyama, T. (1960). Figure-ground dominance as a function of sector angle, brightness, hue, and orientation. Journal of Experimental Psychology, 60, 299-305.

Palmer, S. E., \& Brooks, J. L. (2008). Edge-region grouping in figureground organization and depth perception. Journal of Experimental Psychology: Human Perception and Performance, 34, 1353-1371.

Peterson, M. A., \& Enns, J. T. (2005). The edge complex: Implicit memory for figure assignment in shape perception. Perception \& Psychophysics, 67, 727-740.

Peterson, M. A., Mojica, A. J., Salvagio, E., \& Kimchi, R. (2017). Figural properties are prioritized for search under conditions of uncertainty: Setting boundary conditions on claims that figures automatically attract attention. Attention, Perception, \& Psychophysics, 79, 180199.

Peterson, M. A., \& Salvagio, E. (2008). Inhibitory competition in figureground perception: Context and convexity. Journal of Vision, 8, 113.

Ren, X., Fowlkes, C., \& Malik, J. (2006). Figure/Ground assignment in natural images. European Conference on Computer Vision, 614627.

Rubin, E. (1958). Figure and ground. In D. C. Beardslee \& M. Wertheimer (Eds.) Readings in perception (194-203). Princeton, NJ: Van Nostrand. (Original work published 1915)

Seno, T., Ito, H., \& Sunaga, S. (2009). The object and background hypothesis for vection. Vision Research, 49, 2973-2982.

Takashima, M., Fujii, T., \& Shiina, K. (2012). Face or vase? Areal homogeneity effect. Perception, 41, 1392-1394.

Vecera, S. P., Vogel, E. K., \& Woodman, G. F. (2002). Lower region: A new cue for Figure-Ground assignment. Journal of Experimental Psychology: General, 131, 194-205.

Wagemans, J., Elder, J. H., Kubovy, M., Palmer, S. E., Peterson, M. A., Singh, M., \& von der Heydt, R. (2012). A century of Gestalt psychology in visual perception: I. Perceptual grouping and figureground organization. Psychological Bulletin, 138, 1172-1217.

Wake, H., Wake, T., \& Oyama, T. (2014). Rotating goblet and talking profiles: Does a rotating goblet increase the figural dominance of profiles in Rubin's type of figure-ground reversal patterns? Perception, 43, 1018-1032.

Zhou, H., Freidman, H. S., \& von der Heydt, R. (2000). Coding of border ownership in monkey visual cortex. Journal of Neuroscience, 20, 6594-6611. 